

\title{
Terrain modelling with multifractional Brownian motion and self-regulating processes
}

Antoine Echelard, Olivier Barrière, Jacques Lévy Véhel

\section{To cite this version:}

Antoine Echelard, Olivier Barrière, Jacques Lévy Véhel. Terrain modelling with multifractional Brownian motion and self-regulating processes. ICCVG 2010, Sep 2010, Warsaw, Poland. pp.342-351. inria-00538907

\section{HAL Id: inria-00538907 https://hal.inria.fr/inria-00538907}

Submitted on 24 Nov 2010

HAL is a multi-disciplinary open access archive for the deposit and dissemination of scientific research documents, whether they are published or not. The documents may come from teaching and research institutions in France or abroad, or from public or private research centers.
L'archive ouverte pluridisciplinaire HAL, est destinée au dépôt et à la diffusion de documents scientifiques de niveau recherche, publiés ou non, émanant des établissements d'enseignement et de recherche français ou étrangers, des laboratoires publics ou privés. 


\title{
Terrain modelling with multifractional Brownian motion and self-regulating processes
}

\author{
Antoine Echelard, Olivier Barrière, and Jacques Lévy-Véhel \\ Institut National de Recherche en Informatique et Automatique \\ Parc Orsay Université, 4 rue J. Monod, 91893 Orsay Cedex France \\ \{antoine.echelard, olivier.barriere, jacques.levy-vehel\}@inria.fr
}

\begin{abstract}
Approximate scale-invariance and local regularity properties of natural terrains suggest that they can be a accurately modeled with random processes which are locally fractal. Current models for terrain modeling include fractional and multifractional Brownian motion. Though these processes have proved useful, they miss an important feature of real terrains: typically, the local regularity of a mountain at a given point is strongly correlated with the height of this point. For instance, young mountains are such that high altitude regions are often more irregular than low altitude ones. We detail in this work the construction of a stochastic process called the Self-Regulated Multifractional Process, whose regularity at each point is, almost surely, a deterministic function of the amplitude. This property makes such a process a versatile and powerful model for real terrains. We demonstrate its use with numerical experiments on several types of mountains.
\end{abstract}

Key words: Digital elevation models, Hölderian regularity, (multifractional) Brownian motion.

\section{Motivation and background}

A digital elevation model (DEM) is a digital representation of ground surface topography or terrain. DEM are widely used for geographic information systems and for obtaining relief maps. In most places, the surface of the earth is rough and rapidly varying. 2D random processes are thus often used as models for DEM. In addition, most terrains possess a form of approximate self-similarity, i.e. the same features are statistically observed at various resolutions. For these reasons, fractal stochastic processes are popular models for DEM.

The most widely used set of models is based on fractional brownian motion ( $\mathrm{fBm}$ ) and its extensions. fBm has been used for mountain synthesis as well as in the fine description of the sea floor [PP98]. One of the reasons for the success of fBm is that it shares an important property of many natural grounds: statistically, a natural ground is the same at several resolutions. $\mathrm{fBm}$ allows to model this scale-invariance property as well as to control the general appearance of the ground via a parameter $H$ taking values in $(0,1): H$ close to 0 translates into an irregular terrain, while $H$ close to 1 yields smooth surfaces. More precisely, one can show that, almost surely, the local regularity of the paths of $\mathrm{fBm}$, as measured by the Hölder exponent (see definition 22), is at any point equal to $H$. A large body of works has been devoted to the synthesis and estimation of $\mathrm{fBm}$ from numerical data.

A major drawback of $\mathrm{fBm}$ is that its regularity is the same at every point. This does not fit into reality: for example, erosion phenomena will smooth parts of a mountain more than others. Multifractional Brownian motion $(\mathrm{mBm})$ goes beyond $\mathrm{fBm}$ by allowing $H$ to vary in space. Multifractional Brownian motion simply replaces the real parameter $H$ of $\mathrm{fBm}$ with a function, still ranging in $(0,1)$, with the property that, at each point $(x, y)$, the Hölder exponent 
of a realization is $H(x, y)$. $\mathrm{mBm}$ therefore makes it possible to model a terrain which is smooth at parts and irregular at others, or to synthesize an region containing both a plain and a mountain.

While $\mathrm{mBm}$ does improve on $\mathrm{fBm}$ for DEM modelling, it does not allow to account in a simple way for the following natural fact: typically, altitude and regularity, in a given terrain, are correlated. For instance, in young mountains, regions at low altitudes are generally smoother than regions at high altitudes: indeed, we have evidenced empirically a link between regularity and altitude on various mountainous zones (see below). Moreover, the sign of the observed correlation allows, to a certain extent, to classify mountains according to their erosion degree. This seems to indicate that mountains should be modeled through a new kind of process, where amplitude and regularity are linked, rather than as an $\mathrm{mBm}$, where the regularity is tuned independently from the amplitude. The purpose if this work is to introduce the Self-Regulating Multifractional Process (SRMP) as a means to do so.

This remainder of this paper is structured as follows: section 2 describes in detail the technical aspects of $\mathrm{fBm}, \mathrm{mBm}$ and SRMP and includes simulations that allow one to form an idea of the behaviour of these processes. Section 3 presents a method for estimating the parameters. We present in section 4 the results of the modeling by an SRMP of mountain images that were obtained from the United States Geological Survey site. Finally, section 5 recapitulates the obtained results and gives prospects for future research.

\section{Several models for natural terrains}

\subsection{Fractional brownian motion}

Definition and first properties Fractional brownian motion was introduced by Kolmogorov in 1940 [Kol40] and further studied by Mandelbrot and Van Ness in [MVN68]. It has proved a useful model in many applications, including financial mathematics [Sot03], synthesis of artificial mountains or submarine floors [PP98] or the study of Internet traffic [MN97].

$\mathrm{fBm}$ is a Gaussian centered process with stationary increments. It is parametrized by a constant $H$. It is self-similar, which means that, denoting the process $B_{H}(t), B_{H}(\alpha t)$ follows the same law as $\alpha^{H} B_{H}(t)$ for every fixed $\alpha>0$. A formal definition is as follows:

Definition 21 The bidimensional isotropic fractional Brownian motion with Hurst parameter $H$ is the centered Gaussian process $B_{H}$ such that, for every $(x, y) \in \mathbb{R}^{2}$ :

$$
\mathbb{E}\left(B_{H}(\boldsymbol{x}) B_{H}(\boldsymbol{y})\right) \propto\|\boldsymbol{x}\|^{2 H}+\|\boldsymbol{y}\|^{2 H}-\|\boldsymbol{x}-\boldsymbol{y}\|^{2 H},
$$

where $\|$.$\| denotes the Euclidean norm. Fractional Brownian motion is an extension of the ordi-$ nary Brownian motion: for $H=1 / 2$ it reduces to the well-known Wiener process.

Regularity properties fBm is a continuous but almost surely non-differentiable process. Its regularity can be more precisely measured with the help of the pointwise Hölder exponent, whose definition we recall now in a particular case that will be of interest for us.

Definition 22 Let $\alpha \in(0,1)$. Let $f$ be a function from $\mathbb{R}$ to $\mathbb{R}$ and $x_{0} \in \mathbb{R}$.

$f \in C^{\alpha}\left(x_{0}\right)$ if there exists $\epsilon>0$ and $C \in \mathbb{R}$ such that $\forall t,|t|<\epsilon:\left|f\left(x_{0}+t\right)-f\left(x_{0}\right)\right| \leq C|t|^{\alpha}$.

The pointwise Hölder exponent of $f$ at $x_{0}$ is $: \alpha_{f}\left(x_{0}\right)=\sup \left\{\alpha \in(0,1): f \in C^{\alpha}\left(x_{0}\right)\right\}$.

This exponent may be roughly understood as follows: the largest increments $f(x)-f\left(x_{0}\right)$ in the neighbourhood of $x_{0}$ are of the order of $\left|x-x_{0}\right|^{\alpha_{f}\left(x_{0}\right)}$.

The parameter $H$ of fractional Brownian motion allows to control its regularity in the sense of Hölder exponent. Indeed, almost surely, for all $t, \alpha_{B_{H}}(t)=H$. A a consequence, the higher $H$, the smoother are the trajectories. Figure 1 illustrates this remark. 


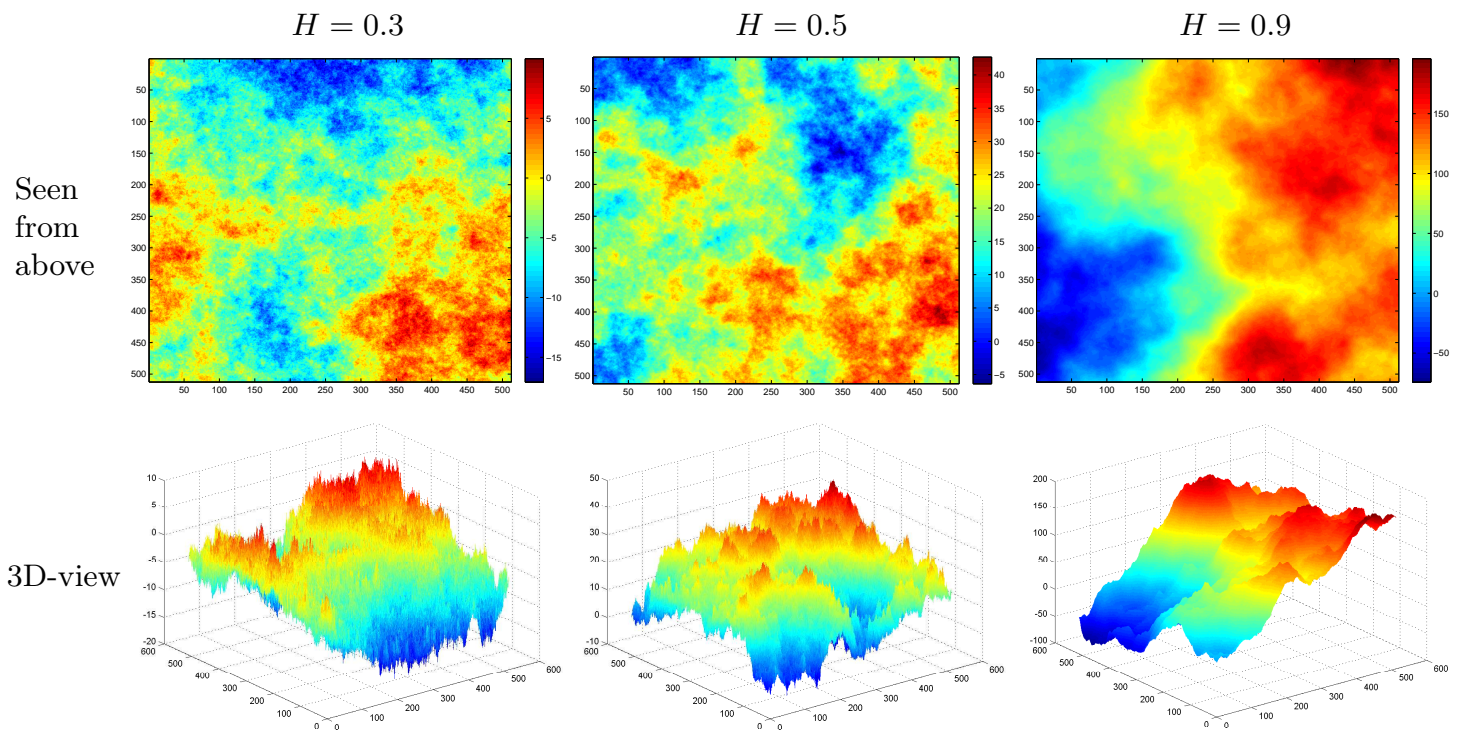

Fig. 1. Realisations of a fractional brownian motion with Hurst parameter $H$

\subsection{Multifractionnal Brownian motion}

Motivation and definition As mentioned above, an important drawback of fBm for terrain modeling is its constant regularity. Indeed, erosion and meteorological phenomena, for instance, smooth some parts of real terrains more than others. More generally, it is obvious that the earth surface presents a variable regularity. This evolving regularity has important consequences in various applications and should be taken into account by a proper model. Multifractional brownian motion, introduced in [PLV95,BJR97], allows to describe such a behaviour.

Definition 23 Let $H$ be a continuous function from $\mathbb{R}^{2}$ to $\mathbb{R}$. The multifractional Brownian motion is the centered Gaussian process with covariance function:

$$
\mathbb{E}\left(B_{H(.)}(\boldsymbol{x}) B_{H(.)}(\boldsymbol{y})\right) \propto\|\boldsymbol{x}\|^{H(\boldsymbol{x})+H(\boldsymbol{y})}+\|\boldsymbol{y}\|^{H(\boldsymbol{x})+H(\boldsymbol{y})}-\|\boldsymbol{x}-\boldsymbol{y}\|^{H(\boldsymbol{x})+H(\boldsymbol{y})}
$$

Regularity properties As in the case of the fractional Brownian motion, we use the Hölder exponent to measure the regularity of the trajectories. Assume that $H$ is a differentiable function. Then one may show that almost surely, for all $(x, y): \alpha_{B_{H(x, y)}}(x, y)=H(x, y)$.

Figure 2 illustrates the variable regularity property of $\mathrm{mBm}$, with a trajectory obtained for a logistic $H$ function. $\mathrm{mBm}$ is a quite versatile model. It allows to deal with numerous phenomena, at the expense of more mathematical complexity. As explained in the previous section, a further generalization is needed in order to model images where the regularity depends on the amplitude of the process, rather than being tuned exogenously.

\subsection{Self-regulating multifractional process}

The motivation for introducing self-regulating processes is based on the following general fact: "in nature, the local regularity of a phenomenon is often related to its amplitude". In addition 


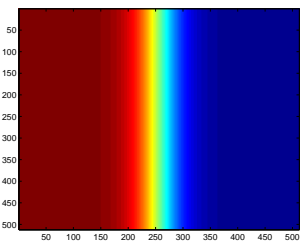

Logistic function $H$ (seen from above)

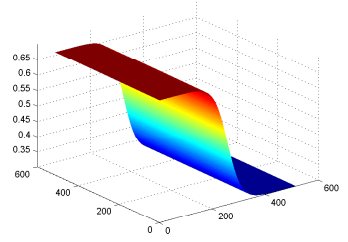

Logistic function $H$ (3D-view)

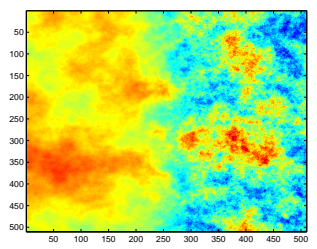

$\mathrm{mBm}$ with parameter $H$ (seen from above)

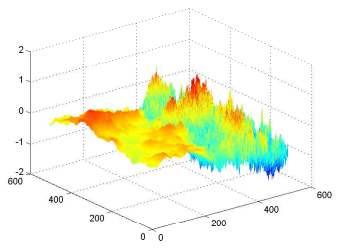

$\mathrm{mBm}$ with parameter $H$ (3D-view)

Fig. 2. A realisation of a $2 \mathrm{D}-\mathrm{mBm}$ with functional parameter $H$



Himalaya



Sub-image

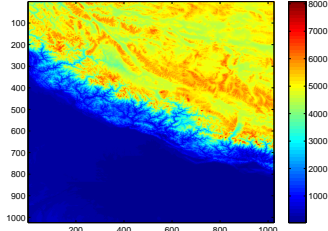

Matlab view

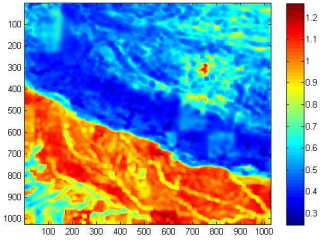

Hölder exponents

Fig. 3. Zones of high altitude in Himalaya have smaller regularity than regions of lower altitude.

to natural terrains, we have verified this fact experimentally on temperatures records and on RR intervals extracted from ECG [Bar07,BLV08]. Figure 3 exemplifies this on a part of Himalaya.

A self-regulating process is by definition a stochastic process such that, almost surely, at each point, the Hölder exponent is a deterministic function of the amplitude. In other words, almost surely, at every point $x, \alpha_{Z}(x)=g(Z(x))$ where $\alpha_{Z}$ is the Hölder exponent of $Z, Z$ the process (e.g., the altitude) and $g$ a deterministic function characterizing the process. There are many ways to design self-regulating processes. A natural one is to modify $\mathrm{mBm}$ so as to obtain the desired property [Bar07]. This process is called the self-regulating multifractional process (SRMP). As figure 4 shows, the SRMP allows to synthesize realistic terrains. On these landscapes, the "plain" areas (with low altitude) are "automatically" smoother than "mountainous" areas (with high altitude) through a specific choice of the function $g$.

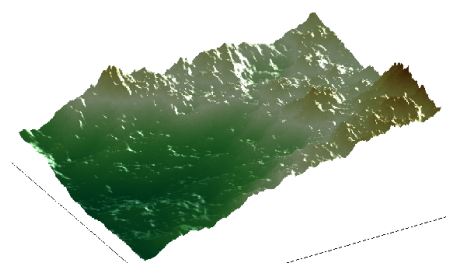

Fig. 4. A realisation of a bidimensional self-regulated multifractional process with $g(Z)=(1-Z)^{2}$ Construction Technically, the SRMP is build using a bidimensional Gaussian field of fractional Brownian motions (introduced in [AT04]). This field, denoted $\{B(H, x, y)\}_{(H, x, y) \in[a, b] \times[0,1]^{2}}$ is defined on $[a, b] \times[0,1]^{2}$ where $\left.[a, b] \subset\right] 0,1[$.

For any non-constant continuous $f$ function defined on a compact set $K \subset \mathbb{R}^{2}$ and ranging in $\mathbb{R}$, and any two reals $\alpha$ et $\beta$, we denote: $\bar{f}_{\alpha}^{\beta}=\alpha+(\beta-\alpha) \frac{f-\inf _{K}(f)}{\sup _{K}(f)-\inf _{K}(f)}$.

Definition 24 Let $g$ be a $k$-Lipschitz function defined on $[\alpha, \beta]$ with values $:[a, b] \subset(0,1)$. 
Let $\alpha^{\prime}$ and $\beta^{\prime}$ be two random variables such that $\alpha \leq \alpha^{\prime}<\beta^{\prime} \leq \beta$. The stochastic operator $\Lambda_{\alpha^{\prime}, \beta^{\prime}}$ from $\mathcal{C}\left([0,1]^{2},[\alpha, \beta]\right)$ into itself is defined almost surely as:

$\Lambda_{\alpha^{\prime}, \beta^{\prime}}(Z)=\underline{\overline{B g(Z)}_{\alpha^{\prime}}} \beta^{\prime}$ where $B_{g(Z)}$ is the function $(x, y) \mapsto B_{g(Z(x, y))}(x, y)$.

Proposition 21 Almost surely, $\Lambda_{\alpha^{\prime}, \beta^{\prime}}$ has a unique fixed-point, that is, there exists exactly one function $(x, y) \rightarrow Z^{*}(x, y)$ such that $: Z^{*}=\underline{\overline{B_{g\left(Z^{*}\right)}} \alpha^{\prime}} \alpha^{\prime}$

Definition 25 The self-regulating multifractional bidimensional process (SRMP2D) parametrized by the function $g$ is defined almost surely as the only fixed-point $Z^{*}$ of $\Lambda_{\alpha^{\prime}, \beta^{\prime}}$.

Regularity properties of the SRMP The following important property is shown in [Bar07]:

Theorem 1. Let $Z_{g}$ be an SRMP2D. Almost surely, $\forall(x, y) \in[0,1]^{2}: \alpha_{Z_{g}}(x, y)=g\left(Z_{g}(x, y)\right)$

In order to use the SRMP2D as a model for real terrains, it is necessary to estimate the function $g$ from numerical data. This is the topic of the next section.

\section{Evaluation of the Hölder exponent}

The method for estimating the local regularity is based on the so-called generalized quadratic variations [IL97]. More precisely, we have used the technique presented in [ALV04], which we briefly recall. Consider a discretized $\mathrm{mBm} \tilde{B}$ parameterized by a function $H$ with values in $[a, b] \subset(0,1)$. Choose $\delta, \gamma$ such that $\delta-\gamma>\frac{1}{4}$ and $\gamma>\delta b$.

Definition $31 \tilde{V}_{N}(t)=\sum_{p \in \tilde{\nu}_{N}(t)}\left(\sum_{k \in F} d_{k} \tilde{B}\left(\frac{p+k}{N^{\delta}}\right)\right)^{2}$ where $t=\left(t_{1}, t_{2}\right)$ and $\tilde{\nu}_{N}(t)=\tilde{\nu}_{N}^{1}\left(t_{1}\right) \times$ $\tilde{\nu}_{N}^{2}\left(t_{2}\right)$ and for $i=1,2: \tilde{\nu}_{N}^{i}\left(t_{i}\right)=\left\{p_{i} \in \mathbb{N} ; 0 \leq p_{i} \leq N-2\right.$ and $\left.\left|t_{i}-\frac{p_{i}}{N^{\delta}}\right| \leq N^{-\gamma}\right\}$.

Theorem 2. Almost surely : $\lim _{N \rightarrow \infty} \frac{1}{2 \delta}\left(2(1-\gamma)-\frac{\log \tilde{V}_{N}(t)}{\log N}\right)=H(t)$.

This theorem gives an algorithm to estimate the exponent of an $\mathrm{mBm}$ that is discretized at locations $t_{i j}=(i / N, j / N), i, j=0, \ldots, N-1$ (we denote $\tilde{B}\left(t_{i j}\right)$ its values): one first computes $\tilde{V}_{N}\left(t_{i j}\right)$ for every $t_{i j}$ and then evaluate its exponent at point $t_{i j}$ with: $\hat{H}\left(t_{i j}\right)=\frac{1}{2 \delta}\left((1-\gamma)-\frac{\log \tilde{V}_{N}\left(t_{i j}\right)}{\log N}\right)$.

This method suffers from the following drawback. Assume $\tilde{B}$ is multiplied by a constant $G$. Then $\tilde{V}_{N}($.$) is multiplied by G^{2}$ and an offset appears: $\Delta_{G}=H_{G B}-H_{B}=-\frac{\log G}{\delta \log N}$

In practice, $N$ has a finite value and the offset of the estimator $\frac{\log G}{\log N}$ can be non-negligible. It is possible to design techniques that correct this offset [Bar07], but we will not use them here. Indeed, what we are looking for is mainly a relation between the exponent and the altitude. The existence of such relation is not affected by this offset. One must however keep in mind that, in the following, the exponents are evaluated within an additive constant.

Theorem 2 shows that this method is theoretically justified in the case of an $\mathrm{mBm}$ : if the resolution is good enough then the estimator will be close to the true Hölder exponent.

We now turn to our problem, i.e. checking for the existence of a relation $g$ between exponents and altitude on images. To estimate this function $g$, we proceed as follows: 
1. The pointwise Hölder exponent is evaluated at each point of the image. We use the method described above under the assumption that the signal is an SRMP. In this case, contrarily to the situation of $\mathrm{mBm}$, it has not been proved that it gives the correct result. However, numerical experiments on simulated SRMP have shown that it seems to behave well.

2. We draw a scatter plot in the "phase plane", that is the altitude-Hölder exponent plane: each point $(x, y)$ of the image with altitude $Z(x, y)$ and estimated regularity exponent $\hat{\alpha}_{Z}(x, y)$ is drawn as a point with coordinates $\left(Z(x, y), \hat{\alpha}_{Z}(x, y)\right)$ in this plane.

If a relation $\alpha_{Z}=g(Z)$ exists, then every point in the phase plane should approximately belong to the graph of the function $g$. In order to improve the readability of the scatter plot, a plot of the histogram is drawn instead: the plane (altitude, exponent) is divided into squares whose color becomes "hotter" as more points belong to the square. To test the method, we applied it to an actual SRMP defined by the function $g(Z)=1-Z$. As one can see it on figure 5 , the points obtained are, within an offset, very close to the graph of the function. This suggests that the previous scheme, although crude, allows to detect relationships between exponents and altitudes.

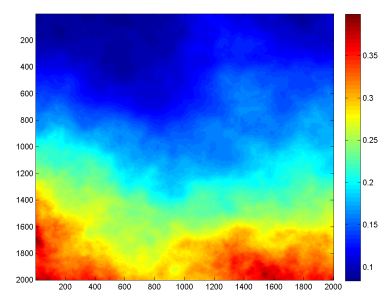

A realization of the SRMP

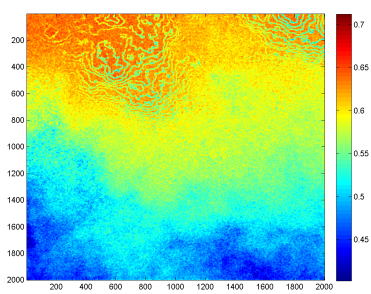

Its exponent at each point

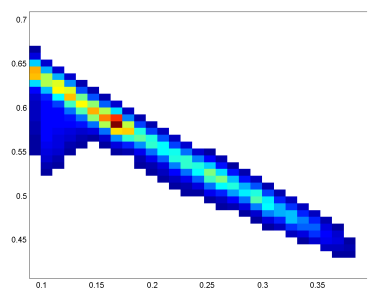

Density of the points in the "exponent-altitude" plane

Fig. 5. The SRMP and its evaluated exponents are related.

\section{Study of mountain images}

\subsection{Study of global images}

We have studied four mountain images. Two of them are "young" mountains: Himalaya and Rocky Mountains. The other two are "old" mountains: Massif Central (France) and Tibesti (Chad). The data were obtained from the internet site of the United States Geological Survey. Their resolution is 3 arc seconds (approx. $90 \mathrm{~m}$ ). They have the following dimensions: Tibesti: 6097x6393; Massif Central: 3807x3835; Rocky Mountains: 9274x6072; Himalaya: 5620x6767.

In order to check for a possible relation of the type $\alpha=g(z)$, we represent as explained above the scatter diagram in the "altitude-exponent" plane. However, the results are not satisfying. Indeed, very scattered exponents with the same altitude are observed. As a consequence, the points can not fit the graph of a function. Rather, roughly elliptic "blobs" are observed. See figure 6 . This tends to indicate that very large grounds are not homogeneous enough to be represented by an SRMP. In order to find such a relation, we now study subsets of the images.

\subsection{Research on sub-images}

A correlation coefficient with a high absolute value shows the existence of an affine relationship that is verified in a approximate way. For this reason, we have been looking for sub-images of 


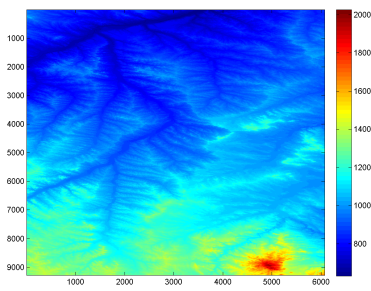

Original image

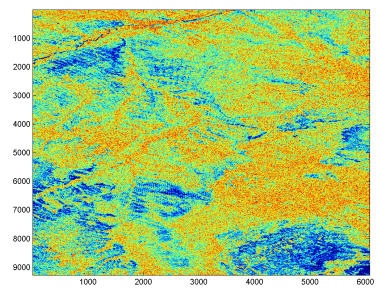

Estimated exponents

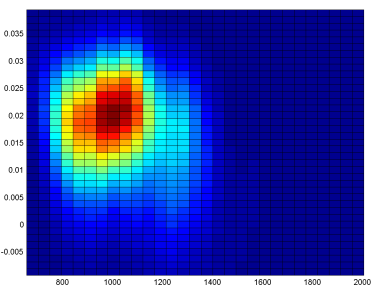

Density of the scatter plot in the "exponent-altitude" plane

Fig. 6. Rocky Mountains

the original images on which the correlation coefficient between the Hölder exponent and the altitude is high. In that view, a square window of size $256^{2}$ or $512^{2}$ pixels was moved over each of the four previous images by steps of 50 pixels. The correlation between the Hölder exponent and the altitude was computed on each sub-image.

On many windows, the correlation is significant. For images with side of length 256 pixels, the percentage of correlation coefficients with higher absolute value that 0.6 are $3.6 \%, 23.4 \%, 10.7 \%$ et $3.9 \%$ respectively for Tibesti, Himalaya, Massif Central and Rocky Mountains. For images with side of length 512 , these proportions are respectively $1.7 \%, 16.6 \%, 8.9 \%$ and $1.7 \%$. Figure 7 gives an example for each mountain and window size 512. The results were similar with size 256 .



Massif central
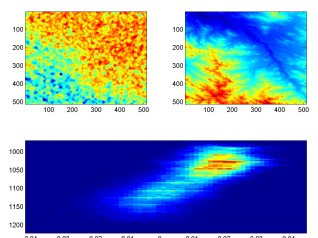

Rocky mountains
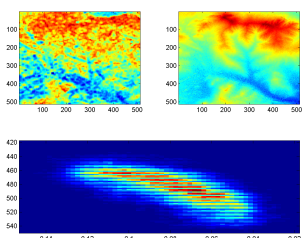

Tibesti
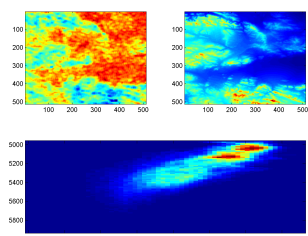

Himalaya

Fig. 7. In each cell, the upper-right figure is the original sub-image of length 512 pixels, the upper-left figure displays the exponent at each point of the sub-image and the lower figure shows the density of the scatter plot in the phase plane.

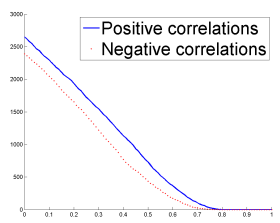

Massif central

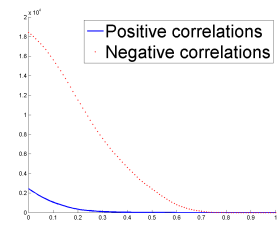

Rocky Moutains



Tibesti

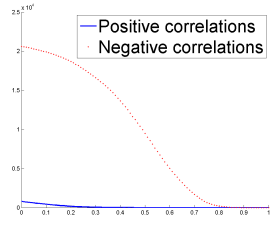

Himalaya

Fig. 8. For a given abscissa $x$, in red, the number of windows with a correlation higher than $x$ and in blue, the number of windows with a correlation smaller than $-x$. For instance, the blue circle on the Tibesti figure means: "2600 windows have a correlation smaller than -0.1 ". 
Moreover, on both young mountains, negative correlations are much more frequent than positive correlations. This means that higher peaks are craggier. To quantify this observation, we plot on figure 8 the following graphs: in red, the number of windows with a correlation higher than $x$ and in blue, the number of windows with a correlation that is smaller than $-x$ (this is for $256^{2}$ images. Again, similar results are obtained for $512^{2}$ ones). The absence of significant positive correlations between exponent and altitude appears to be characteristic of young mountains. Indeed, on both young mountains, the few positive correlations are not significant: less than 0.4. The situation is reversed for older mountains, probably because of erosion: high-altitude surfaces of old mountains are smoothed out by erosion, and thus high altitudes translates into more smoothness. For Tibesti, positive correlations are predominant. For Massif Central, the situation is balanced : approximately as many positive correlations as negative ones are observed.

\section{Conclusion and perspectives}

We have been looking for a relation between Hölder exponent and altitude. This does not seem to occur for large pieces of land, but significant correlations are observed on smaller parts of terrains. This suggests the existence of a linear relation between exponents and altitude.

Moreover, negative correlations are largely predominant in sub-images from young-mountain images whereas positive significant correlations occur on old mountains. Indeed, the peaks of young mountains are craggier than plains whereas high-altitude surfaces of old mountains are smoothed out by erosion. This property allows to distinguish between young and old mountains.

Because of the rough way of estimating the exponent, we have only been looking for linear relationships. In the future, it would be interesting to estimate more finely both this relationship and the regularity exponents. This would allow to model in a more satisfying way natural terrains as realizations of SRMP.

\section{References}

ALV04. A. Ayache and J. Lévy-Véhel. Identification of the pointwise holder exponent of generalized multifractional brownian motion. Stoch. Processes and their Applications, 111:119-156, 2004.

AT04. A. Ayache and M.S. Taqqu. Multifractional processes with random exponent. Stoch. Processes and their Applications, 111(1):119-156, 2004.

Bar07. O. Barrière. Synthèse et estimation de mouvements browniens multifractionnaires et autres processus à régularité prescrite. Définition du processus autorégulé multifractionnaire et applications. PhD thesis, IRCCyN, 2007.

BJR97. A. Benassi, S. Jaffard, and D. Roux. Elliptic Gaussian random processes. Rev. Mat. Iberoamericana, 13(1):19-90, 1997.

BLV08. O. Barrière and J. Lévy-Véhel. Local regularity analysis of rr intervals. In CBMS, 072008.

IL97. J. Istas and G. Lang. Quadratic variations and estimation of the local hölder index of a gaussian process. Ann. Inst. H Poincaré 4 (33), pages 407-436, 1997.

Kol40. A.N. Kolmogorov. Wienersche spiralen und einige andere interessante kurven im hilbertschen raume. Doklady, 26:115-118, 1940.

MN97. P. Mannersalo and I. Norros. Multifractal analysis of real ATM traffic: a first look. 1997.

MVN68. B. Mandelbrot and J.W. Van-Ness. Fractional brownian motion, fractional noises and applications. Siam Review, pages 422-437, 1968.

PLV95. R.F. Peltier and J. Lévy-Véhel. Multifractal brownian motion : definition and preliminary results. Technical report, INRIA, 1995.

PP98. B. Pesquet-Popescu. Modélisation bidimensionnelle de processus non stationnaires et application à l'étude du fond sous-marin. PhD thesis, ENS de Cachan, 1998.

Sot03. T. Sottinen. Fractional Brownian motion in finance and queueing. $\mathrm{PhD}$ thesis, University of Helsinki, 2003. 